\title{
Imaging and Microanalysis of Iron Biominerals
}

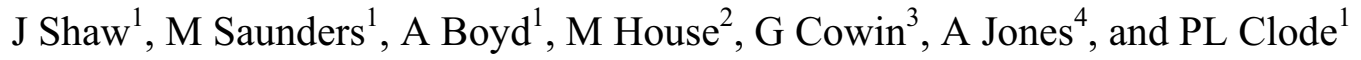 \\ ${ }^{1 .}$ Centre for Microscopy, Characterisation and Analysis, The University of Western Australia, Perth, \\ Australia. \\ 2. Biomagnetics Group, The University of Western Australia, Perth, Australia. \\ 3. Centre for Advanced Imaging, University of Queensland, Brisbane, Australia. \\ 4. School of Medical and Molecular Biosciences, University of Technology Sydney, Sydney, Australia.
}

Iron plays a critical role in the metabolic processes of virtually all life on Earth. Specifically, biological systems have harnessed the oxidation-reduction capacity of iron, which can cycle between its ferrous $\left(\mathrm{Fe}^{2+}\right)$ and ferric $\left(\mathrm{Fe}^{3+}\right)$ states with relative ease, thereby driving a range of reactions within the body. In addition to its role in metabolism, certain animals exploit iron's other physical properties for various structural and functional purposes. For example, certain molluscs reinforce their teeth with iron to feed on rocks [1] and some animals are thought to use iron particles similar to those found in magnetotactic bacteria to sense magnetic fields [2]. Here we show that correlative imaging and microanalytical tools can be used to identify, map and characterise biological iron deposits across a range of spatial scales.

\section{Iron biominerals in mollusc teeth}

Biological systems produce a range of iron minerals. Commonly, organisms store iron as ferritin, which comprises a ferrihydrite core within a protein cage. This mineral phase is known to act as an amorphous precursor for the subsequent formation of other iron oxide phases, including lepidocrocite, goethite and magnetite. Chitons are marine molluscs with teeth that exemplify this process, and each of these minerals can be detected within various architecturally discrete regions that provide the teeth with unique functional and mechanical properties. Using a combination of techniques from X-ray micro-CT through to SEM and TEM we have investigated all aspects of the biomineral system from the overall architecture to the composition and crystallography at the nanoscale (see Figures 1 and 2).

\section{Iron containing cells in the honeybee abdomen}

Shortly after hatching from the comb, worker honeybees begin the process of accumulating iron within fat cells (trophocytes) in the abdomen. The reason for this is unclear, but links to iron metabolism, ballast for flight and even magnetic navigation have been suggested. Micro-MRI has been used to reveal the distribution of iron in bee trophocytes. We are using this, and other correlative imaging methods, to search for similar iron deposits in bee body parts such as the head and antennae, which have also been postulated to contain a magnetic sensory system.

\section{Summary}

Understanding the structure and composition of iron biominerals is important for resolving the mechanisms involved in their formation and elucidating their biological function. Correlative imaging and analytical modalities that bridge macro to nanometre length scales can resolve the complex structural hierarchy of biominerals and may aid in the discovery and characterization of an iron based magnetoreceptor system in honeybees and other animals. For more information on the analysis of potential iron-containing magnetoreceptors see Boyd, et al elsewhere at this conference [3]. 


\section{References:}

[1] M Saunders et al, Journal of Structural Biology 167 (2009), 55-61.

[2] CD Treiber et al, Nature 484 (2012), 367-370.

[3] The authors acknowledge the facilities, scientific and technical assistance of the Australian Microscopy \& Microanalysis Research Facility at the Centre for Microscopy, Characterisation \& Analysis, The University of Western Australia, a facility funded by the University, State and Commonwealth Governments.

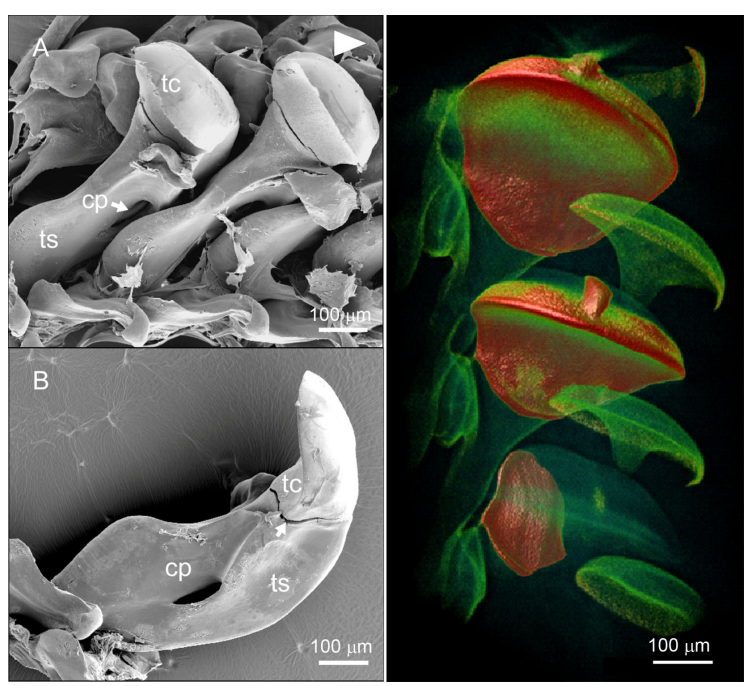

Figure 1. SEM (A and B left) and micro-CT (right) have been used to image overall tooth structure to link the internal mineral architecture with tooth function. Red and green regions denote iron and organic rich regions, respectively.

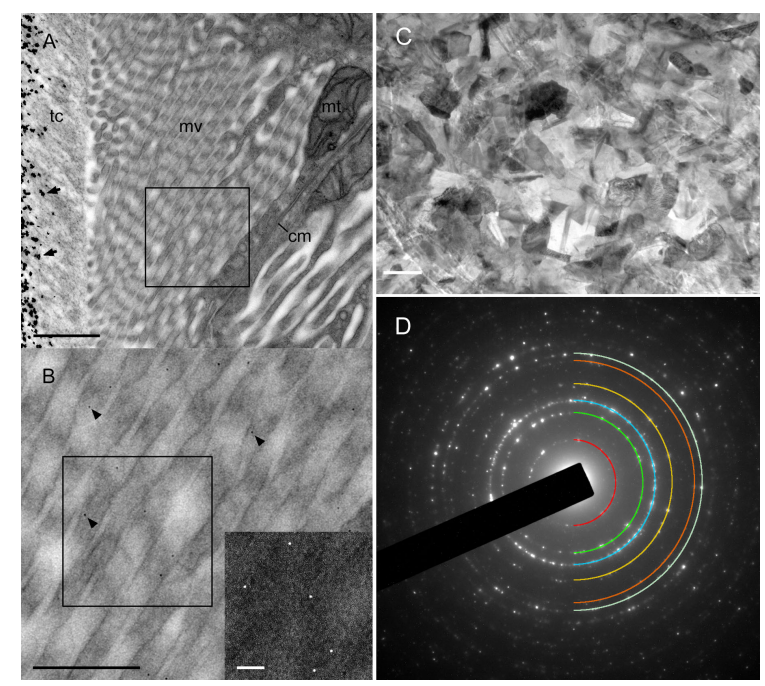

Figure 2. TEM and EFTEM of the cells surrounding the tooth cusps (A and B) show that electron dense particles (arrows) match 7-8 $\mathrm{nm}$ iron rich particles consistent with ferritin (inset). Focused ion beam methods have also been used to produce TEM sections of mineralised teeth (C) and electron diffraction has been used to characterise the mineral phases (D). 